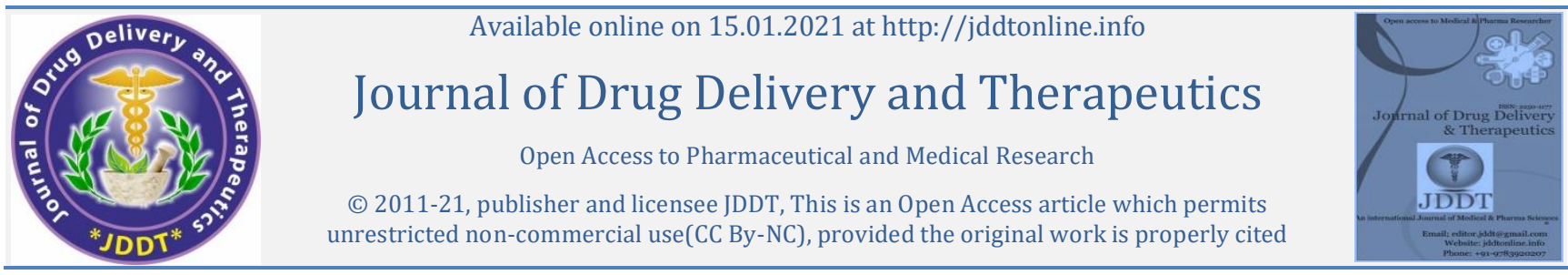

Open Access Full Text Article

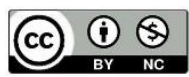

Review Article

\title{
A Systematic Review on Lamotrigine Induced Skin Rashes
}

\author{
T.T. Muhammed Anwar*, P.P. Muhamed Faris, Thansiha \\ Department of Pharmacy Practice, College of Pharmaceutical Sciences, Government Medical College, Kozhikode, Kerala, 673008, India
}

Article Info:

Article History:

Received 11 Oct 2020;

Review Completed 28 Nov 2020

Accepted 09 Dec 2020;

Available online 15 Jan 2021

Keywords:

Anti-epileptic drugs; Lamotrigine; Rashes; Seizure

Cite this article as:

Anwar TTM, Faris PPM, Thansiha, A Systematic Review on Lamotrigine Induced Skin Rashes, Journal of Drug Delivery and Therapeutics. 2021; 11(1):146-151 DOI: http://dx.doi.org/10.22270/jddt.v11i1.4633

*Address for Correspondence:

T.T. Muhammed Anwar, Department of Pharmacy

Practice, College of Pharmaceutical Sciences,

Government Medical College, Kozhikode,

Kerala,673008, India
Abstract

Lamotrigine is a newer anti-epileptic drug synthesised in 1980s and approved by USFDA in 1994. It is mainly used for treating paediatric epilepsy and one of the frequently prescribing newer anti- epileptic drugs in European countries. In English scenario, lamotrigine is suggested as monotherapy for recently identified partial seizures (localized seizure) in children as its first line drug. Lamotrigine is recommended for generalized seizures as second line drug and as add on therapy in intractable generalized epilepsy. It is a treatment option after sodium valproate and ethosuximide for petit-mal epilepsy. It can be used as either single or in combination with other anti-epileptic drugs. It has lot of adverse effects like other anti-epileptic drugs. Major adverse effect associated with lamotrigine is skin rashes in newly administered patients up to 8 weeks. About $3-10 \%$ of the patients experience skin rashes in their therapy with lamotrigine. Lamotrigine doses should be titrated properly in combination with other anti-epileptic drugs especially with valproic acid. Other less common adverse effects with lamotrigine include dizziness, ataxia, blurred vision, headache, nausea and diplopia. Moreover, lamotrigine has a safe drug profile and effective in both children and adults for the treatment of various types of seizures. The dose escalation should be monitored regularly to avoid serious adverse reactions.

\section{Introduction}

Lamotrigine is a newer antiepileptic drug synthesised in 1980s and approved by United States Food and Drug Administration (USFDA) in 1994. It is mainly used for treating paediatric epilepsy and it is one of the most frequently prescribing newer anti-epileptic drugs in European countries. In English scenario, lamotrigine is suggested as monotherapy for recently identified partial seizures (localized seizure) in children as its first line drug. Lamotrigine is recommended for generalized seizures as second line drug and as add on therapy in intractable generalized epilepsy. It is a treatment option after sodium valproate and ethosuximide for petit-mal epilepsy. It can be used either as single or in combination with other antiepileptic drugs. ${ }^{1}$

If lamotrigine is administering with other anti-epileptic drugs, then its dose should be strictly based upon the alongside drug. The administration of LTG (Lamotrigine) with phenobarbital, carbamazepine, phenytoin or oxcarbazepine result in an enhanced LTG clearance and cause a significant reduction in plasma drug concentration, therefore a higher dose is recommended. While considering its administration with valproic acid, a lower dose is recommended as valproate shows a considerable hoist in blood stream concentration of LTG by lowering its clearance. LTG causes minor skin rashes to severe condition such as
Stevens-Johnson's syndrome in children. Adults are less prone to skin rashes than children. Other common ADRs include neurological abnormalities which are mainly depending upon the dose. ${ }^{2}$

Lamotrigine blocks slow inactivated sodium channels and increases the effective refractory period. It can act by unchanging the typical synaptic conduction and maintain a normal refractory period in the spinal cord of mammals. It can also regulate the calcium channel conductance. LTG produce type IV hypersensitivity reactions usually after four weeks of starting dose and suddenly reverse the condition on discontinuation of therapy. It shows eosinophilia, muscle pain, lymph edema and malaise along with rashes. The patients who are initially taking valproic acid, on add on therapy with LTG are more susceptible to rashes. A marginal increase from low initial dose can reduce this risk. ${ }^{3}$

\section{Materials and Methods}

Data were collected from various research articles, case studies and review articles containing details regarding lamotrigine, adverse effects of lamotrigine and adverse effects of other anti-epileptic drugs. Electronic databases such as Google Scholar and PubMed were also used for data collection. 


\section{Results}

A study on the evaluation of tolerability and effectiveness of lamotrigine in infants having a partial seizure describes both new patients as well as patients having a history of lamotrigine treatment achieve better therapeutic outcomes and tolerability. The drug dose was regulated in accordance with the previous exposure with lamotrigine and the adjuvant anti-epileptic therapy. Among these patients, about $10 \%$ moved to monotherapy of lamotrigine. There wasn't any case reported with serious skin rashes. Commonly reported ADR includes pyrexia, infection to ear and URT. ${ }^{4}$

A multi-centered study participated children below thirteen years old from eleven countries to determine the tolerability and efficacy of lamotrigine, showed a better seizure control especially in atonic seizure and absence seizure. It also showed a significant effect on other types of seizures. Dose was adjusted in accordance with the adjunctive therapy. In combination with valproate, lamotrigine is given as $1-5 \mathrm{mg} /$ $\mathrm{kg}$ once a day whereas in absence of valproate it can be given as $5-15 \mathrm{mg} / \mathrm{kg}$ every day. The most common adverse events include skin rashes, drowsiness, sleepiness and exacerbation of seizures etc. The withdrawal rate was about $12.6 \%$ because of its adverse events. 5

Lamotrigine was used as adjunctive therapy in infants in their first life year with partial seizure, infantile spasm or intractable seizures. Seizures are better controlled during the first three months of the therapy. Clearance of seizure was observed during the first life year. Lamotrigine has age depended kinetics, better efficacy and well tolerated. ${ }^{6}$

In the case of refractory epilepsy, lamotrigine in combination with valproate or divalproex sodium was very effective. 1-2 years of follow up in the paediatric population with refractory epilepsy showed a significant benefit of the therapy. Skin rash was common and led to the withdrawal of the therapy. The adverse events can be reduced and the treatment efficacy can be improve by introducing lamotrigine in lower doses. 7

A study was conducted in Shaheed Sadoughi hospital (Iran) to evaluate the safety and efficacy of lamotrigine as an adjuvant therapy for one of the most burdensome seizures (Lennox-Gastaut syndrome) in refractory seizure of children between the age group of 2-6. It was an empirical interventional study comprised of 40 subjects (22 male and 18 female) organized in 2007. Follow up of the therapy was done after 3 months and the result showed that $12 \%$ of the subjects were free of epilepsy. There was a significant reduction $(>50 \%)$ in epileptic frequency among $52 \%$ of the subjects, but $12 \%$ of subjects showed an increase in epilepsy. It indicates that the drug was efficacious in both generalized and partial seizures. $12.5 \%$ of the subjects showed short term side effects like ataxia and drowsiness. There were no serious side effects in any of the subjects. The study concluded that lamotrigine can be given as an adjuvant therapy for controlling refractory epilepsy with LennoxGastaut syndrome. ${ }^{8}$

An open labeled study was conducted to evaluate the long term use of lamotrigine in paediatric patients as monotherapy and adjuvant therapy for the treatment of various types of seizures including focal seizure, petit mal seizure and Lennox-Gastaut syndrome. 252 subjects below 16 years of age for a duration of 48 weeks to 144 weeks were enlisted in the study. The clinical status of subjects were examined in every 24 hebdomads throughout the therapy and the average time period of lamotrigine exposure was 96.7 hebdomads. $9.1 \%$ of the subjects showed dizziness and $6.3 \%$ showed somnolence. Other common adverse drug reactions include headache, nausea and vomiting. There was a significant improvement in clinical status of $75 \%$ of subjects during the treatment period of 48-96 hebdomads. Serious adverse reactions reported in these subjects were infection and pneumonia. The study concluded that monotherapy or add on therapy of lamotrigine was effective and tolerated over a period of two years in paediatric patients having both generalized and partial seizures. The positive result of the study suggests that lamotrigine has clinical benefits in adults for both short term and long term therapy. ${ }^{9}$

A randomized control study determines the long term safety profile and the possible drug interaction of a total of 3783 paediatric ( $\leq 18$ years) patient under lamotrigine monotherapy. Among the total patients about 2222 were affected by adverse events. The most commonly reported ADR was skin rash $(7.3 \%)$ and the rare one was the StevensJohnson syndrome. $1.9 \%$ of the patients were withdrawn from therapy due to an adverse drug reaction. Treatment withdrawal was mainly due to variety of skin rashes and secondly because of the increased seizures. Patients on monotherapy have a lower risk of developing adverse drug reaction as compared to polytherapy. ${ }^{10}$

Lamotrigine was given as adjuvant therapy in an open trial in children with intractable seizures. The study was conducted over a period of seven months duration consisting of 37 participants. The study subjects were divided into two groups; 1) patients on valproate 2) patients on nonvalproate. Patients in first and second groups were given $5 \mathrm{mg}$ and $15 \mathrm{mg} / \mathrm{kg}$ of lamotrigine respectively. $21.6 \%$ of the subjects cured completely by the treatment and there was a significant reduction $(>50 \%)$ in $13.5 \%$ of patients. More than $50 \%$ of the patients did not show any changes in their condition and $10 \%$ experienced a worsened disease condition. This study revealed that lamotrigine is tolerated in children and does not produce serious side effects in the same. It can be used in the absent and atonic epileptic conditions. ${ }^{11}$

A prospective study was conducted to evaluate the adverse effect and efficacy of lamotrigine in children having epilepsia major. 56 subjects were enrolled in the study and produced a notable result, in which $11 \%$ of the subjects produced complete cure in their conditions and $43 \%$ of them seemed to $>50 \%$ of reduction in epileptic frequency. For the treatment of epilepsia major, lamotrigine is an effective campaigner. $20 \%$ of subjects having Lennox-Gastaut syndrome showed a complete cure and $53 \%$ of them produce a marked improvement. There were no serious side effects with lamotrigine monotherapy. When given along with valproate, $9 \%$ of the subjects suffered with rashes and $12 \%$ of them experienced an increase in epileptic frequency. This study concludes that lamotrigine is an effective drug for the management of epilepsia major in children. ${ }^{12}$

A study was conducted in patients having age in between 219 to find out long term efficacy, safety and tolerability of lamotrigine in controlling seizure. 155 subjects were given lamotrigine alone or as an adjuvant therapy up to 4 years. The treatment was spanned over 53-221 hebdomads and produced a significant epileptic control. It indicates the possibility of long term maintenance of epileptic control than a short term treatment plan. A marked improvement in alertness, quality of life, seizure severity, behavior and mobility were shown in 19 patients with lamotrigine therapy. Adverse events related to lamotrigine show a mild intensity and only $4 \%$ of the subjects were terminated from the investigation. 13 
Efficacy and tolerability of lamotrigine monotherapy were studied in children having petit mal epilepsy which were newly diagnosed. A sample size of 54 was selected and conducted a twenty four hour confirmatory EEG. Then study was conducted for a period of 20 weeks in which lamotrigine dose was titrated up to $10.2 \mathrm{mg} / \mathrm{kg}$ reached or epilepsy was managed. The epilepsy control data were evaluated using EEG and found that control of seizure occurred in the fifth week of the therapy. The common side effects include cough and headache. It indicates that lamotrigine monotherapy is effective and well tolerated in petit mal seizures in children. ${ }^{14}$

An observational study was conducted in 11316 seizure patients for evaluating the safety and effectiveness of lamotrigine using a prescription event monitoring system. A follow-up was done in 3994 patients with 36 months of lamotrigine therapy. The result of the study concluded that, the most common side effect was rashes in $1.97 \%$ of patients and $2 \%$ of the subjects discontinued the therapy due to side effects during first month of therapy. Children in the age group 2-12 years were more prone to rashes than adults. There were several other adverse events reported with lamotrigine which include drowsiness, vomiting, lassitude, headache, nausea and malaise. There were some rare effects like neutropenia, thrombocytopenia, Stevens-Johnson syndrome, leucopenia, acute renal failure, meningitic reactions, lupus like reaction and hepatotoxicity. There were no events reported with lamotrigine when used in pregnant women and no deaths were reported with lamotrigine therapy. This study concluded that lamotrigine therapy is effective and safe when used in patients with intractable seizures. 15

A dose titrating study succeeded by double blind placebo controlled study was conducted to evaluate the safe and effective use of lamotrigine monotherapy in newly detected petit mal seizure in children under the age group of 3-15 years. About 44 subjects were enrolled and EEG was used for evaluating the treatment outcome. The safety of the treatment was assessed by laboratory, physical and neurological examinations, vital signs and adverse events. In the first dose titrating study, $74.1 \%$ of the subjects became epilepsy free and in double blind placebo controlled period a statistically significant number of subjects (62\%) were epilepsy free than treatment with placebo(21\%). The study concluded that treatment with lamotrigine alone is safe, effective and tolerated in patients with petit mal epilepsy. ${ }^{16}$

A survey was conducted over different countries of Europe for assessing the quality of life of patients with epilepsy and to find out the effect of therapy on their seizure control. Over 5000 subjects were participated in the survey using a questionnaire administered by members of seizure assist team. Most of the subjects were not satisfied with this treatment and they thought that therapy is not enough for their seizure control. Most of them denounced these situations. This study concluded that, in the management of epilepsy importance should be given not only to seizure control but also to the improvement in the quality of life of the patient. ${ }^{17}$

528 patients treated with anti-epileptic drugs were enrolled in a study for a period of 1 year using the case records obtained from a consulting neurologist. The study found that grand mal epilepsy and focal impaired-awareness seizures were the most common types and it is frequently shown in male patients. Most patients were treated with combination therapy of several anti-epileptic drugs and mild to moderate side effects were shown mainly in female patients. ${ }^{18}$
A systematic review was conducted to identify the safety and ADRs of lamotrigine in children below 18 years using different research articles available in the PubMed, EMBASE, Cochrane library and MEDLINE. From 78 articles, about 3783 subjects were recognized. 2222 adverse drug reactions were reported and major was rashes in $7.3 \%$ of the patients. Steven- Johnson syndrome was rarely seen in some patients and about $1.9 \%$ of the subjects discontinued therapy due to adverse reactions. The study concluded that patients administering lamotrigine monotherapy were more susceptible to rashes. The patients who are taking combination therapy have more chances of adverse reactions than monotherapy. ${ }^{19}$

A randomized, double blind controlled clinical trial was conducted in 453 children with petit mal seizures to evaluate the safety, efficacy and tolerability of lamotrigine, ethosuximide and valproic acid. Doses of drugs were titrated until subjects were seizure free, maximum tolerated dose reached or when treatment failure occurred. Attention deficit disorder was more seen with valproic acid. There were no significant differences in between discontinuation of therapy due to adverse effects among these 3 drugs. The study concluded that ethosuximide and valproic acid were more effective than lamotrigine in petit mal seizure. ${ }^{20}$

A study was conducted in adolescents and children of sample size 30 with generalized seizures to find out the effect of lamotrigine as an adjuvant therapy with other anti-epileptic drugs, the subjects already taking. It was a placebo controlled, double blind cross over study. Out of 30 subjects, 20 of them had Lennox-Gastaut syndrome. Lamotrigine concentrations were individualized anteceding the placebo treatment. The result showed a significant difference between the effects of lamotrigine and placebo treatment. They concluded that lamotrigine is a tolerated and effective treatment option with other anti-epileptic drugs for the treatment of refractory seizures. ${ }^{21}$

An open trial was conducted to compare the efficacy and safety of lamotrigine and carbamazepine for the treatment of localized seizures. 417and 201 subjects were enrolled in lamotrigine and carbamazepine group respectively. Dose titration was conducted in the first six hebdomads of the therapy and maintenance dose was calculated according to the response of the subjects. The results showed that only lesser numbers of subjects experienced adverse effects and discontinued the therapy in lamotrigine group compared to carbamazepine.The most common adverse effects include rashes and somnolence. It concluded that both carbamazepine and lamotrigine were effective in the treatment of localized seizures. But lamotrigine was more tolerable than carbamazepine. ${ }^{22}$

A randomized, open label, cross over study was conducted for assessing the effect of lamotrigine in 18 patients, who were receiving sodium valproate $500 \mathrm{mg}$ twice a day. The subjects received valproate in optimum therapeutic concentration. Lamotrigine was administered in 3 different doses of 50, 100 and $150 \mathrm{mg}$ eventually with a washout period of two weeks between the doses. There was a significant reduction in clearance of lamotrigine and an increase in half life when combined with valproate. It indicates that a reduction in dose of lamotrigine is recommended when given along with valproate. ${ }^{23}$

A review article studied adverse events with lamotrigine therapy by analyzing 13 different studies conducted in 1096 children with various types of seizures. It showed that lamotrigine monotherapy is effective and produces less adverse events compared to combination therapy with other drugs. Major adverse events were rashes in children and its 
incidence increases when given along with sodium valproate. Other AEs include nausea, ataxia, dizziness, diplopia, tremor and urinary tract infections. The study concluded that adverse events like rashes with lamotrigine can be reduced by proper dosing and dose titration. ${ }^{24}$

In another study, the efficacy and safety of lamotrigine was evaluated by comparing adjuvant therapy and placebo. 201 subjects (adolescents and children) were selected in this study with localized seizures, who were taking other antiepileptic drugs. The study was conducted for 6 hebdomads of dose titration and 12 hebdomads of dose maintenance period. There was a significant reduction in seizure frequency in lamotrigine group compared to placebo. Vomiting, infection and somnolence were seen both in placebo and lamotrigine group. But dizziness, nausea, ataxia and tremor were more frequent in LTG group. 2 of the subjects experienced skin rashes and cured after discontinuation of LTG therapy. The study concluded that lamotrigine showed prominent effectiveness, safety and tolerability. It can be given as combination therapy with other anti-epileptic drugs for the management of focal or localized seizures in children. 25

A retrospective study was conducted in Columbia in outpatients for assessing the occurrence of rashes associated with lamotrigine. The sample size of the study was 988 . Their result explained that, $5.7 \%$ of the patients experienced rashes and $3.9 \%$ of them discontinued the therapy due to this reason. One of the patients suffered Steven-Johnson syndrome and none of them were hospitalized due to severe rash conditions. Patients under 13 years of age are more prone to lamotrigine induced skin rashes. They concluded that, if the patient had a history of another anti-epileptic drug induced rashes, they are more susceptible to lamotrigine induced rashes. ${ }^{26}$

A study was conducted in China to find out the relationship between genetics and lamotrigine induced cutaneous ADRs. They selected 43 patients with lamotrigine induced adverse reactions and checked whether any link between HLA-B*1502 and lamotrigine induced cutaneous reactions. The study concluded that there was no significant relation between these items and a larger study with more sample size is needed to obtain a considerable result. ${ }^{27}$

A randomized, placebo controlled, blinded study was conducted to determine the tolerability and efficacy of combination therapy of lamotrigine with other anti-epileptic drugs for the treatment of grand mal epilepsy of patients having more than 2 years of age. 45 subjects from 2-20 years of age group were selected and the study period was 12 hebdomads for subjects between the age of 2-12 years and 7 hebdomads for patients older than 12 years. No cases of rashes reported from both placebo and LTG groups and one of the patients with lamotrigine therapy experienced disorientation. The study concluded that lamotrigine adjuvant therapy with other AEDs was effective in subjects between the age of 2-20 years for the treatment of grand mal epilepsy. 28

There were many adverse drug reactions reported along with the usage of anti-epileptic drugs. Some of the reactions are predictive and some of them are idiosyncratic. The clinical condition, lab data, dose titration and risk factors should be analyzed before administering these drugs to the patient. Nowadays genotype analysis is conducted to assess the relationship between genetics and antiepileptic drug induced adverse reactions. Close monitoring of these factors can reduce ADRs and it will improve the quality of life of the patient. 29
The tolerability and efficacy of lamotrigine combination therapy with other anti-epileptic drugs were analyzed by randomized, placebo controlled, double blind study in children having less than 2 years of age with focal seizures. The sample size was 177 and time period of the study was 8 hebdomads. There was a better seizure control produced by lamotrigine group compared to placebo. This indicated the efficacy and safety profile of lamotrigine in children with less than 2 years having localized seizures. ${ }^{30}$

An open label, randomized parallel design was conducted to compare between lamotrigine and valproic acid on their efficacy in adolescents and children with petit mal seizure. 38 subjects were selected between the age of 3 and 13 years and equally divided into two groups; one taking lamotrigine and other taking valproic acid. Both drugs were started with a minimum dose and titrated according to guidelines up to a maximum safe concentration obtained or seizure free condition occurred. The effect of drug therapy was evaluated by an electroencephalogram. $52.6 \%$ and $5.3 \%$ of the participants were seizure free after 1 month from valproic acid and lamotrigine group respectively. It indicated that both drugs were effective in petit mal seizure treatment and valproic acid has a much quicker onset of action compared to lamotrigine. ${ }^{31}$

A study was conducted in UK by prescription event monitoring(PEM) for the post marketing surveillance of lamotrigine in an epilepsy centre and compared with other newer drugs such as vigabatrin and gabapentin for adverse drug reactions. 2701 subjects were enrolled in the study who were exposed to lamotrigine and other two newer drugs. The most common adverse event with lamotrigine was skin rashes. Some of them experienced renal failure, intravascular coagulation and hepatic failure. No one in the subjects died due to LTG induced ADR. Study showed that lamotrigine has a reliable safety profile and very rarely shows, dangerous adverse drug reactions. ${ }^{32}$

A case report explained about the adverse event of lamotrigine in breastfed infant. The infant showed episodes of severe asphyxia and the reason behind this was mother took lamotrigine throughout the pregnancy. LTG passed to the infant through breast feeding and attained a blood concentration of 4.87 micrograms per ml. There are several studies that conclude the relationship between lamotrigine and infant asphyxia.The breastfeeding mother should be aware about her medications during pregnancy and postpartum state. 33

All AEDs showed lots of cutaneous adverse drug reactions. Lamotrigine produced skin rashes in 3-10 \% of the patients. Combination therapy with valproic acid, previous rashes history with other anti-epileptic drugs are risk factors for LTG induced rashes. Some of the patients experienced epidermal necrolysis with adjuvant therapy with lamotrigine and valproic acid. The physicians should be alert about the doses of lamotrigine that cause adverse reactions and its combination with other anti-epileptic drugs. ${ }^{34}$

Lamotrigine is one of the newer anti-epileptic drugs. The common adverse reactions with lamotrigine include dizziness, ataxia, skin rashes, blurred vision, headache, nausea and diplopia. Some of the patients experience organ failures such as hepatic and renal failure. ${ }^{35}$

A case study reported drug reaction with eosinophilia and systemic symptoms (DRESS) syndrome. The lamotrigine was the reason behind this situation. The patient was admitted with absence seizures and given lamotrigine therapy. After 45 days of treatment, the patient showed fever and rashes. Then patient was confirmed with acute pancreatitis. It was 
the first case, reported with lamotrigine induced DRESS syndrome. 36

A review report explained about one of the common antiepileptic drugs, lamotrigine and its adverse effects. The most common adverse effects include skin rashes which mainly occur in the first 8 weeks of the therapy. About $10 \%$ of the patients experienced lamotrigine induced skin rashes. Some of the patients suffered from serious rashes and were hospitalized. Pediatric patients and Adjunct therapy with valproate were the most common risk factors for lamotrigine induced skin rashes. ${ }^{37}$

The combination of valproic acid with lamotrigine increases the half-life of lamotrigine up to 3 times. A prospective study was conducted to evaluate the effect of addition of lamotrigine into valproic acid containing drug regimen. 108 subjects were enrolled, in which 30 of them were, with generalised seizure, 60 with localized seizure and 12 with Lennox Gastaut syndrome. In 37 subjects, lamotrigine was added to monotherapy of valproic acid and in 71 of the subjects with valproic acid and other anti-epileptic drugs. The study subjects were monitored for 27 months or up to lamotrigine withdrawal from the regimen. They added lamotrigine in $80 \%$ of the patients and discontinued from $20 \%$ of the subject's regimen. Major reason for discontinuation was rashes. There were other adverse reactions which include dizziness, insomnia, fatigue, headache and GI symptoms. Other serious adverse reactions occurred with some of the patients include hepatotoxicity, hallucination and irritability. This concluded that the addition of lamotrigine into valproic acid containing drug regimen is acceptable with side effects. The addition of lamotrigine into a dosage regimen is applicable with proper titration of doses. ${ }^{38}$

\section{Conclusion}

Lamotrigine is a newer anti-epileptic drug. It has a lot of adverse effects like other anti-epileptic drugs. Major adverse effect associated with lamotrigine is skin rashes in newly administered patients for up to 8 weeks. About 3-10 \% of the patients experience skin rashes in their therapy with lamotrigine. Dose of lamotrigine should be titrated properly if used in combination with other anti-epileptic drugs, especially with valproic acid. Other less common adverse effects with lamotrigine include dizziness, ataxia, blurred vision, headache, nausea and diplopia. Moreover, lamotrigine has a safe drug profile and effective in both children and adults for the treatment of various types of seizures. The dose escalation should be monitored regularly to avoid serious adverse reactions.

\section{References}

1. Egunsola O, Choonara I, Sammons HM. Safety of lamotrigine in paediatrics: a systematic review. BMJ open. 2015 Jun 1; 5(6):e007711.

2. Wong IC, Mawer GE, Sander JW. Factors influencing the incidence of lamotrigine-related skin rash. Ann Pharmacother. 1999 Oct; 33(10):1037-42.

3. Matsuo F. Lamotrigine. Epilepsia. 1999 May; 40:s30-6.

4. Piña-Garza JE, Elterman RD, Ayala R, Corral M, Mikati MA, PiñaGarza MJ, Warnock CR, Conklin HS, Messenheimer JA. Long-term tolerability and efficacy of lamotrigine in infants 1 to 24 months old. Jchild neurol. 2008 Aug; 23(8):853-61.

5. Besag FM, Wallace SJ, Dulac O, Alving J, Spencer SC, Hosking G. Lamotrigine for the treatment of epilepsy in childhood. J pediatrics. 1995 Dec 1; 127(6):991-7.

6. Mikati MA, Fayad M, Koleilat M, Mounla N, Hussein R, Kazma A, Yunis K. Efficacy, tolerability, and kinetics of lamotrigine in infants.Jpediatrics. 2002 Jul 1; 141(1):31-5.
7. Thome-Souza S, Valente KD. Valproate and lamotrigine in pediatric patients with refractory epilepsy: after the first year. Pediatr Neurol. 2013 Jun 1; 48(6):436-42.

8. Falah R, Akhavan KS, Golestan M. Efficacy and safety of lamotrigine in lennox-Gastaut syndrome. Iranian Journal of Child Neurology, Vol. 3 No. 3 (2009).

9. Duchowny M, Gilman J, Messenheimer J, et al. Long-term tolerability and efficacy of lamotrigine in pediatric patients with epilepsy. J Child Neurol 2002; 17:278-85.

10. Egunsola O, Choonara I, Sammons HM. Safety of lamotrigine in paediatrics: a systematic review. BMJ open. 2015 Jun 1; 5(6):e007711.

11. Coppola G, Pascotto A. Lamotrigine as add-on drug in children and adolescents with refractory epilepsy and mental delay: an open trial. Brain and Development. 1997 Sep 1; 19(6):398-402.

12. Farrell K, Connolly MB, Munn R, Peng S, MacWilliam LM. Prospective, open-label, add-on study of lamotrigine in 56 children with intractable generalized epilepsy. Pediatr neurol. 1997 Apr 1; 16(3):201-5.

13. Besag FM, Dulac O, Alving J, Mullens EL. Long-term safety and efficacy of lamotrigine (Lamictal ${ }^{\circledR}$ ) in paediatric patients with epilepsy. Seizure. 1997 Feb 1; 6(1):51-6.

14. Holmes GL, Frank LM, Sheth RD, Philbrook B, Wooten JD, Vuong A, Kerls S, Hammer AE, Messenheimer J. Lamotrigine monotherapy for newly diagnosed typical absence seizures in children. Epilepsy research. 2008 Dec 1; 82(2-3):124-32.

15. Mackay FJ, Wilton LV, Peace GL, Freemantle SN, Mann RD. Safety of long-term lamotrigine in epilepsy. Epilepsia. 1997 Aug; 38(8):881-6.

16. Frank LM, Enlow T, Holmes GL, Manasco P, Concannon S, Chen C, Womble G, Casale EJ. Lamictal (lamotrigine) monotherapy for typical absence seizures in children. Epilepsia. 1999 Jul; 40(7):973-9.

17. Baker GA, Jacoby A, Buck D, Stalgis C, Monnet D. Quality of life of people with epilepsy: a European study. Epilepsia. 1997 Mar; 38(3):353-62.

18. Pal AB, Prusty SK, Sahu PK, Swain TR. Drug utilization pattern of antiepileptic drugs: a pharmacoepidemiologic and pharmacovigilance study in a tertiary teaching hospital in India. Asian J Pharm Clin Res. 2011; 4(1):96-.

19. Egunsola O, Choonara I, Sammons HM. Safety of lamotrigine in paediatrics: a systematic review. BMJ open. 2015 Jun 1; 5(6):e007711.

20. Glauser TA, Cnaan A, Shinnar S, Hirtz DG, Dlugos D, Masur D, Clark PO, Capparelli EV, Adamson PC. Ethosuximide, valproic acid, and lamotrigine in childhood absence epilepsy. N Engl JMed. 2010 Mar 4; 362(9):790-9.

21. Eriksson AS, Nergårdh A, Hoppu K. The efficacy of lamotrigine in children and adolescents with refractory generalized epilepsy: a randomized, double-blind, crossover study. Epilepsia. 1998 May; 39(5):495-501.

22. Nieto-Barrera M, Brozmanova M, Capovilla G, Christe W, Pedersen B, Kane K, O'Neill F, Lamictal vs Carbamazepine Study Group. A comparison of monotherapy with lamotrigine or carbamazepine in patients with newly diagnosed partial epilepsy. Epilepsy research. 2001 Aug 1; 46(2):145-55.

23. Anderson GD, Yau MK, Gidal BE, Harris SJ, Levy RH, Lai AA, Wolf $\mathrm{KB}$, Wargin WA, Dren AT. Bidirectional interaction of valproate and lamotrigine in healthy subjects. Clinical Pharmacology \& Therapeutics. 1996 Aug; 60(2):145-56.

24. Messenheimer JA, Giorgi L, Risner ME. The tolerability of lamotrigine in children. Drug Safety. 2000 Apr 1; 22(4):303-12.

25. Duchowny M, Pellock JM, Graf WD, Billard C, Gilman J, Casale E, Womble G, Risner M, Manasco P, Lamictal Pediatric Partial Seizure Study Group. A placebo-controlled trial of lamotrigine add-on therapy for partial seizures in children. Neurology. 1999 Nov 1; 53(8):1724-.

26. Hirsch LJ, Weintraub DB, Buchsbaum R, Spencer HT, Straka T, Hager M, Resor Jr SR. Predictors of lamotrigine-associated rash. Epilepsia. 2006 Feb; 47(2):318-22.

27. Shi YW, Min FL, Liu XR, Zan LX, Gao MM, Yu MJ, Liao WP. HLA-B alleles and lamotrigine-induced cutaneous adverse drug reactions in the Han Chinese population. Basic \& clinical pharmacology \& toxicology. 2011 Jul; 109(1):42-6.

28. Trevathan E, Kerls SP, Hammer AE, Vuong A, Messenheimer JA. Lamotrigine adjunctive therapy among children and adolescents with primary generalized tonic-clonic seizures. Pediatrics. 2006 Aug 1; 118(2):e371-8. 
29. Zaccara G, Franciotta D, Perucca E. Idiosyncratic adverse reactions to antiepileptic drugs. Epilepsia. 2007 Jul; 48(7):122344.

30. Pina-Garza JE, Levisohn $\mathrm{P}$, Gucuyener K, Mikati MA, Warnock CR, Conklin HS, Messenheimer J. Adjunctive lamotrigine for partial seizures in patients aged 1 to 24 months. Neurology. 2008 May 27; 70(22 Part 2):2099-108.

31. Coppola G, Auricchio G, Federico R, Carotenuto M, Pascotto A. Lamotrigine versus valproic acid as first-line monotherapy in newly diagnosed typical absence seizures: an open-label, randomized, parallel-group study. Epilepsia. 2004 Sep; 45(9):1049-53.

32. Wong IC, Mawer GE, AS Sander JW. Adverse event monitoring in lamotrigine patients: a pharmacoepidemiologic study in the United Kingdom. Epilepsia. 2001 Feb 8; 42(2):237-44.

33. Nordmo E, Aronsen L, Wasland K, Småbrekke L, Vorren S. Severe apnea in an infant exposed to lamotrigine in breast milk. AnnPharmacother. 2009 Nov; 43(11):1893-7.
34. Kaur S, Dogra A. Toxic epidermal necrolysis due to concomitant use of lamotrigine and valproic acid. IndianJ Dermatol. 2013 Sep; 58(5):406.

35. Sarris BM, Wong JG. Multisystem hypersensitivity reaction to lamotrigine. Neurology. 1999 Oct 1; 53(6):1367-.

36. Roquin G, Peres M, Lerolle N, Dib N, Mercat A, Croue A, Augusto JF. First report of lamotrigine-induced drug rash with eosinophilia and systemic symptoms syndrome with pancreatitis. AnnPharmacother. 2010 Dec; 44(12):1998-2000.

37. Guberman AH, Besag FM, Brodie MJ, Dooley JM, Duchowny MS, Pellock JM, Richens A, Stern RS, Trevathan E. Lamotrigine-associated rash: risk/benefit considerations in adults and children. Epilepsia. 1999 Jul; 40(7):985-91.

38. Faught E, Morris G, Jacobson M, French J, Harden C, Montouris G, Rosenfeld W, Postmarketing Antiepileptic Drug Survey (PADS) Group. Adding lamotrigine to valproate: incidence of rash and other adverse effects. Epilepsia. 1999 Aug; 40(8):1135-40. 